\title{
On a Possible Mechanism of Developing of the Turbulence in Accretion Disks in Nonmagnetic Binary Stars
}

\author{
Dmitry Bisikalo*? Evgeny Kurbatov and Pavel Kaygorodov \\ Institute of Astronomy - RAS, Moscow, Russia \\ E-mail: bisikalo@inasan.ru, kurbatov@inasan.ru, pasha@inasan.ru
}

\begin{abstract}
As well known the Keplerian disks are stable against generation of turbulence. At the other side the astrophysical accretion disks reveal intensive turbulence process which can be identified via relatively high accretion rates. One of the challenging astrophysical problems is the searching for possible instabilities leading to the turbulization of gas-dynamic disks. There are some features that make accretion disks non-keplerian in gas-dynamical flows in binary stellar systems. In particular, the non-symmetric gravitational force causes formation of a precessional wave in the disk. The wave characterized by strong density and velocity gradients. Linear analysis of perturbations in such disks showed that they are subject of small-scale radial instability with growth rate about tenths and hundredths of orbital period of the binary. If the variation of velocity on the perturbation wavelength scale is about or higher than the sound speed, the perturbations become unstable. This instability may lead to growth of the turbulence in one period of the disk. We performed calculations of two disks with different values of the secondary-to-primary mass ratio of binary components, $q=0.05$ and $q=0.27$, and the same separation values. The characteristics of turbulence correspond to observations, namely the Shakura-Sunyaev parameter $\alpha \sim 0.01$. The high- $q$ model showed twice more value of $\alpha$ than the low- $q$ model.
\end{abstract}

XI Multifrequency Behaviour of High Energy Cosmic Sources Workshop

25-30 May 2015

Palermo, Italy

*Speaker.

${ }^{\dagger}$ This work was supported by program RSCF 15-12-30038. 


\section{Introduction}

It is well-known that high accretion rates in the accretion disks of binary stars may be explained only by the turbulent viscosity. Turbulence must be a result of developing instability $[1,2,3]$. Due to the fact that many close binary systems do not possess magnetic fields, we should search for purely gasdynamic instabilities in these systems.

Many researchers have attempted to find a purely gasdynamic instability in the Keplerian accretion disk. (see, e.g. [4]). It is possible to show that radial disturbances are stable in accordance with the Rayleigh criterion [5]. Also Balbus et al. [5] didn't find instabilities in the azimuthal shortwavelength modes of the disturbances. The study of long-wavelength modes in the numerical model of a thin Keplerian disk [4] showed that these disturbances develop up to the non-linear stage and then fade out, which prevents the turbulization of the disk. In [6, 7] the authors supposed that in a disk with the negative gradient of entropy the baroclinic instability can develop in nonaxisymmetric modes. Later Fridman et al. [8] proposed the super-reflection instability as a reason of turbulization. The main disadvantage of these ideas is in the fact that we need to have rather peculiar conditions in the disk to implement them.

There are many various features in the flow pattern of close binary system $[9,10,11,12,13$, 14]. In particular, results of 3-D numerical simulations show that the tidal action of the secondary leads to the formation of special waves in accretion disks, precessional density waves [15]. The wave has spiral structure and propagate over the whole disk. Qualitatively one can imagine precessional wave as an envelope curve of the elliptic orbits that precess in the non-axisymmetric gravitational field. The ellipticity of the orbits may arise from the instability of the eccentricity caused either by viscous forces [16] or tidal resonances [17]. We are mostly interested in how these features can influence to the development of instabilities, turbulization and angular momentum transfer.

In this work we analyze results of study of small perturbations stability in the accretion disks of a close binary system where the spiral precessional density wave exists. Two numerical models were calculated with values of secondary-to-primary mass ratio 0.05 and 0.27 . The detailed description of the method that we use to analyze the disturbances is given in the paper [18].

\section{Precessional density wave}

Gas in inner part of a close binary system undergo the action of the accretor's gravity $\left(a_{\mathrm{a}}\right)$, donor's gravity $\left(a_{\mathrm{d}}\right)$, centrifugal force $\left(a_{\mathrm{c}}\right)$, and pressure $\left(a_{\mathrm{p}}\right)$. Their relative contribution may be estimated as follows:

$$
a_{\mathrm{a}} \sim a_{\mathrm{c}} \gg a_{\mathrm{d}} \sim a_{\mathrm{p}} .
$$

The balance of two main forces, accretor's gravity and centrifugal force, results in the formation of a Keplerian accretion disk. Since $a_{\mathrm{p}} \ll a_{\mathrm{a}}$, gas moves along ballistic trajectories whose shape is close to ellipse. At the same time the non-Keplerian action of the donor star makes elliptical streamlines precess. The precession frequency depends on the characteristic radius of the streamline and grows along with it. The streamlines cannot intersect, hence a quasi-stationary configuration forms. Shape of the precessional wave is governed by the balance of the gravitational influence of the donor-star and the pressure. The results of numerical simulations show that the precessional 
density wave has the form of a strongly twisted spiral and propagates almost the whole disk (see Fig. 1). We may suppose that in the observer's reference frame the precessional density wave rests (the specific time of one cycle is a several tens of orbital periods of the system). Presence of the wave significantly changes the distributions of density, also radial and tangential components of the velocity comparing to the equilibrium Keplerian distributions [15, 18]. In particlar the radial velocity in the wave may vary by several Mach numbers (see Fig. 1).

For this work we've completed calculations of accretion disks in the close binary systems having two values of secondary-to-primary mass ratio (let's designate it $q$ ): 0.05 and 0.27 . Other parameters of the systems (separation $A$, orbital period of the binary $P_{\text {orb }}$ ) are as follows:

\begin{tabular}{c|c|c|c|c}
$q$ & $M_{1}\left[M_{\odot}\right]$ & $M_{2}\left[M_{\odot}\right]$ & $A$ [a.u.] & $P_{\text {orb }}$ [days] \\
\hline 0.05 & 1.0 & 0.05 & 0.0029 & 0.056 \\
0.27 & 0.64 & 0.17 & 0.0032 & 0.075
\end{tabular}

In the high- $q$ disk the binary has a smaller Roche lobe resulting in a $45 \%$ smaller disk (see Fig. 1). As the separation approximately the same in both systems, the high- $q$ disk suffered stronger tidal action from the secondary component. This results in faster precession of the elliptical streamlines, hence a more tight spiral pattern (note the different spatial scales on the plots). Both the density and the velocity components achieve larger values in the high- $q$ model, and hence three-four times larger radial gradients.

\section{Instability of small perturbations in the disk with the precessional wave}

We analyzed small radial perturbations to study effect of non-Keplerian disturbances on the disk stability. As a "non-perturbed" solution for this problem we use the results of numerical simulations described above. The disk is considered to be geometrically thin since the ratio of the disk thickness to it's radius is less than 0.01 . Besides, we can show that perturbations propagating in the $z$ direction (perpendicular to the plane of the disk) are sonic and by default do not result in instabilities. This allows us to significantly simplify our analysis by omitting the vertical degree of freedom. Since the precessional wave is strongly twisted, for every radial direction we can suppose that it is approximately axisymmetric. Thus, we can analyze small perturbations in the frame of the 2D approach independently for every radial direction (for more details see [18]). Presence of the disturbances of the density and velocity distributions in the disks leads to development of the instability of radial modes having specific growth times of tenths and hundredths of the orbital period (see Fig. 2).

Our analysis shows that gradient of the radial velocity plays crucial role in the development of the instability. In the local approach we can write the dispersion relation as [18]:

$$
\omega_{ \pm}=u_{0} k \pm\left[c_{\mathrm{T}}^{2} k^{2}+\varkappa_{0}^{2}-\frac{1}{4}\left(\frac{\partial u_{0}}{\partial r}\right)^{2}\right]^{1 / 2}
$$

where $u_{0}$ is the unperturbed radial velocity, $k$ is the wavenumber, $\varkappa_{0}$ is the epicyclic frequency. The physical sense of this instability is as follows. In the radial Euler equation the term of the radial velocity gradient $\left(\partial u_{0} / \partial r\right)$ acts on a flow element as an external force together with the 

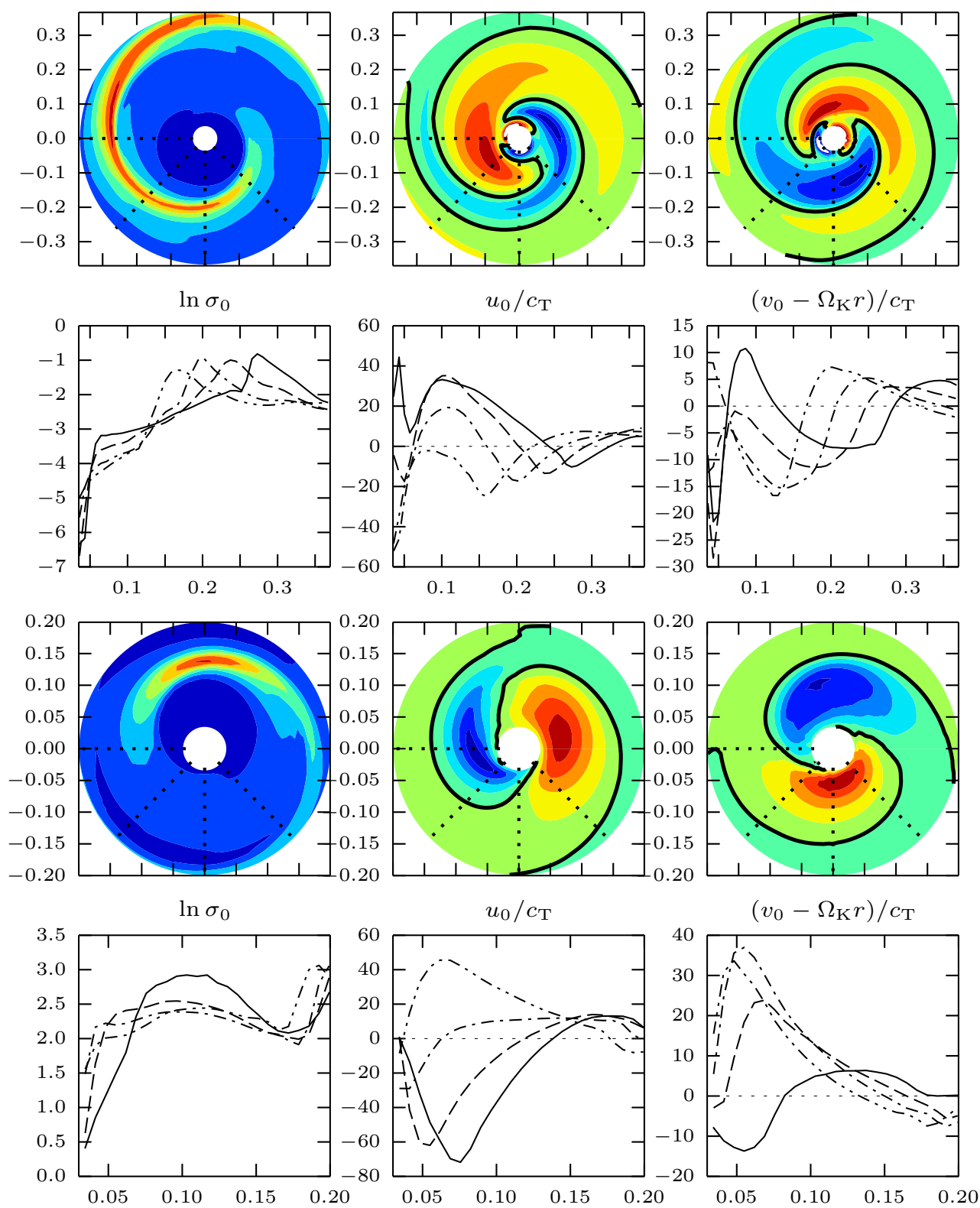

Figure 1: Distributions of the surface density $\left(\sigma_{0}\right.$, left column), radial velocity $\left(u_{0}\right.$, middle column), and azimuthal velocity ( $v_{0}$, right column). Velocities are in units of isothermal sound speed. Each 2-D plot correspond to radial cut on the contour plot above. Two top rows correspond to the model with $q=0.05$ while two bottom rows represent models with $q=0.27$.

pressure and the centrifugal force. In our approach unperturbed rotational flow along with the pressure prevent the development of instability, since they represented by positive terms in under the radical in (3.1). However, if the velocity gradient is sufficiently high, the flux of momentum, transferred to the perturbation, can exceed the contribution of the stabilizing terms. According to (3.1) it happens when the variation of the radial velocity on the perturbation wavelength scale is equal to or exceeds the sound speed. In this case we can state that within one wave period the rear phase of the perturbation is going to catch up with the front phase. In accordance with the mass 
conservation law the amplitude of perturbation maxima grows. In terms of the dispersion relation this means that the non-zero values of the increment must appear.

It is rather obvious however that not all unstable perturbations may result in the development of turbulence in the accretion disk. The conditions for turbulization look as follows: (i) only 3D turbulence can develop; (ii) the specific time of growth of unstable perturbations should not be longer than one disk period. These limitations give us the following local conditions for the wavelength and the value of the increment:

$$
\lambda \leqslant h, \quad \gamma \geqslant \frac{\Omega}{2 \pi} .
$$

The final distributions of the coefficient of turbulent viscosity or the Shakura-Sunyaev parameter over the disk may be given as a weighted sum of the estimates (3.3) for all unstable modes [18].

As was noted above, the model having five times higher secondary-to-primary mass ratio can be characterized by strongly twisted spiral pattern than the low- $q$ model. Since the radial gradients in the high- $q$ disk are three or four times higher, this means accordingly to (3.1) that the instability conditions are less strict in this model. We note also that the high- $q$ disk has the larger velocity variance on the precessional wave. Together with the previous note this may explain why the high- $q$ disk has greater number of the unstable modes of larger increments, than the low- $q$ disk, see Fig. 2.
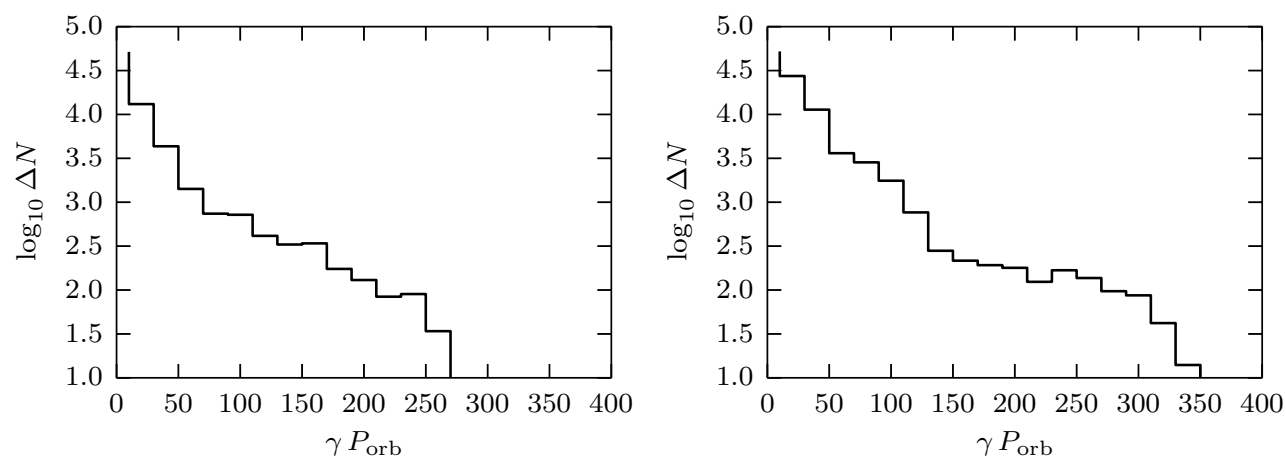

Figure 2: Distribution of the number of modes over positive increments, for two models: $q=0.05$ (left panel) and $q=0.27$ (right panel).

The properties of unstable modes can be connected to characteristics of developed turbulence, in particular to the coefficient of turbulent viscosity $[19,8,20]$ or Shakura-Syunyaev parameter [2]:

$$
v_{\text {turb }}=\frac{\gamma \lambda^{2}}{4 \pi^{2}}, \quad \alpha=\frac{v_{\text {turb }}}{c_{\mathrm{T}} h}
$$

where $\lambda$ and $\gamma$ are the wavelength and increment of perturbations, respectively; $h=c_{\mathrm{T}} / \Omega_{\mathrm{K}}$ is the semi-thickness of an isothermal Keplerian disk.

In Fig. 3 one can see that the Shakura-Sunyaev parameter varies over the disk from 0 to $0.015-$ 0.02 reaching maximal values on the boundaries of the precessional wave where the radial velocity of gas becomes zero (see Fig. 1). The reason of this is in the fact that near zeros of the radial velocity the wavelengths of the modes decrease and as a result most of them meet the first condition of 
turbulization (3.2). The value of the $\alpha$-parameter is higher on the outer boundary of the precessional way, since in the outer region of the disk the second condition of turbulization weakens. We also should note that the model with high secondary-to-primary mass ratio has twice greater value of maximal $\alpha$-parameter. This is the reflection of the fact that the high- $q$ disk undergoes more intensive tidal action from the secondary component than the low- $q$ disk, as was emphasized above.
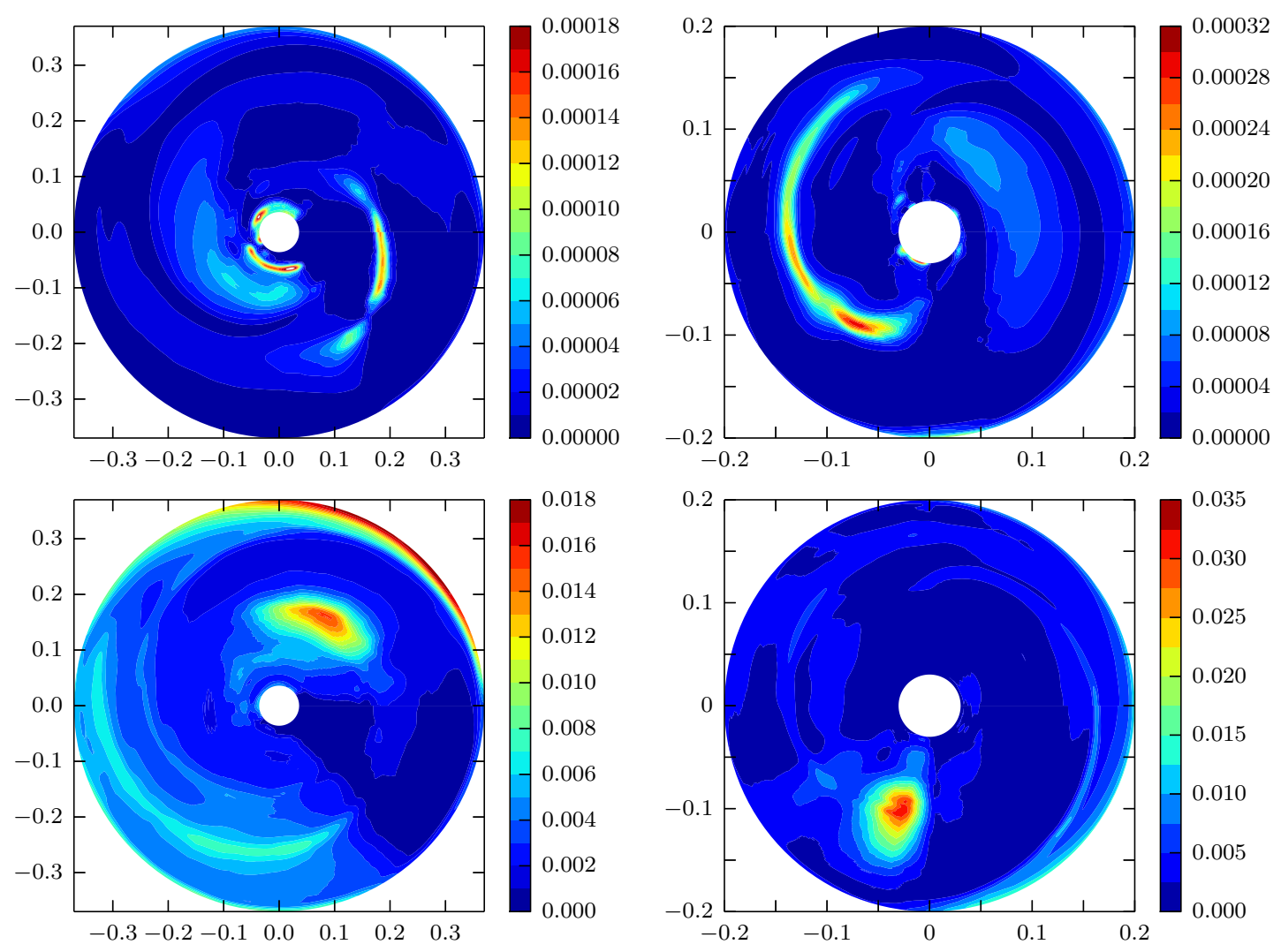

Figure 3: Distributions of the perturbations' amplitude (top row) and Shakura-Sunyaev $\alpha$-parameter (bottom row) for two models: $q=0.05$ (left) and $q=0.27$ (right).

\section{Conclusions}

To develop gas-dynamic instability in an accretion disk we need to have density and velocity distributions significantly different from the Keplerian. An obvious example of this configuration is an accretion disk in the non-axisymmetric gravitational field of a binary star. In [15] authors showed that the gravitational influence of the secondary results in the formation of a precessional density wave in the disk. The wave significantly changes the flow pattern in the disk and causes the appearance of regions with high density and velocity gradients.

In this work we continue to study the stability of accretion disk (started in [18]) with the precessional wave using the method of linear analysis of perturbations. Results of numerical sim- 
ulations of the accretion disk in binary system [15] addressed as the non-perturbed solution. We showed that the presence of the precessional wave gives rise to unstable radial modes having characteristic growth time about hundredths of the orbital period of the binary. It turned out, that the existence of regions with strong gradients of the radial velocity is important for development of the turbulence. The perturbations with wavelength smaller than the disk semi-thickness, growing up to the non-linear stage within one disk cycle, give rise to 3-D turbulence. We performed calculations of two models with different values of the secondary-to-primary mass ratio of binary components, $q=0.05$ and $q=0.27$, but the same separation values. As a consequence of the stronger tidal action in the high- $q$ disk, this model has several times higher radial velocity gradient comparing to the low- $q$ model. Also the variance of the radial velocity over the precessional wave reaches larger values. Meeting both of these trends has lead to the greater maximum value of the Shakura-Sunyaev $\alpha$-parameter in the high- $q$ disk, about 0.035 , compared to 0.018 in the low- $q$ disk.

\section{Acknowledgments}

This work was supported by program RSCF 15-12-30038.

\section{References}

[1] N. I. Shakura, Astron. Zh. 49, 921 (1972).

[2] N. I. Shakura, R. A. Sunyaev, $A \& A$ 24, 337 (1973).

[3] D. Lynden-Bell, J. E. Pringle, MNRAS 168, 603 (1974).

[4] P. Godon, M. Livio, ApJ 521, 319 (1999) [astro-ph/9812082].

[5] S. A. Balbus, J. F. Hawley, J. M. Stone, ApJ 467, 76 (1996).

[6] W. Cabot, ApJ 277, 806 (1984).

[7] H. H. Klahr, P. Bodenheimer, ApJ 582, 869 (2003) [arXiv: astro-ph/ 0211629 ].

[8] A. M. Fridman, D. V. Bisikalo, Phys. Usp. 51, 551 (2008).

[9] K. Sawada, T. Matsuda, I. Hachisu, MNRAS 219, 75 (1986).

[10] K. Sawada, T. Matsuda, I. Hachisu, MNRAS 221, 679 (1986).

[11] K. Sawada, T. Matsuda, M. Inoue, I. Hachisu, MNRAS 224, 307 (1987).

[12] D. V. Bisikalo, A. A. Boyarchuk, O. V. Kuznetsov, V. M. Chechyotkin, Astron. Rep. 41, 786 (1997) [astro-ph/9802004].

[13] D. V. Bisikalo, A. A. Boyarchuk, V. M. Chechetkin, O. A. Kuznetsov, D. Molteni, MNRAS 300, 39 (1998).

[14] D. V. Bisikalo, A. A. Boyarchuk, A. A. Kilpio, O. A. Kuznetsov, Astron. Rep. 45, 676 (2001).

[15] D. V. Bisikalo, A. A. Boyarchuk, P. V. Kaygorodov, O. A. Kuznetsov, T. Matsuda, Astron. Rep. 48, 449 (2004) [astro-ph/ 0403053 ].

[16] Y. E. Lyubarskij, K. A. Postnov, M. E. Prokhorov, MNRAS, 266, 583 (1994).

[17] S. H. Lubow, ApJ 381, 259 (1991). 
[18] E. P. Kurbatov, D. V. Bisikalo, P. V. Kaygorodov, Phys. Usp. 57, 851-863 (2014) [arXiv:1409.8492].

[19] V. M. Canuto, I. Goldman, O. Hubickyj, ApJ (Lett.) 280, L55 (1984).

[20] B. B. Kadomtsev, in Review of plasma physics, edited by M. A. Leontovich (New York (NY, USA): Consultants Bureau (Plenum)), vol. 4 (1966). 